MATHEMATICS OF COMPUTATION

Volume 78, Number 266, April 2009, Pages 1227-1230

S $0025-5718(08) 02168-6$

Article electronically published on August 20, 2008

\title{
NEW SUMS OF THREE CUBES
}

\author{
ANDREAS-STEPHAN ELSENHANS AND JÖRG JAHNEL
}

\begin{abstract}
We report on our search for solutions of the Diophantine equation $x^{3}+y^{3}+z^{3}=n$ for $n<1000$ and $|x|,|y|,|z|<10^{14}$.
\end{abstract}

\section{INTRODUCTION}

It is a long standing problem as to whether every rational integer $n \not \equiv 4,5$ $(\bmod 9)$ can be written as a sum of three integral cubes. According to the web page [Be of Daniel Bernstein, the first attacks by computer were carried out as early as 1955 .

Nevertheless, for example, for $n=3$, there is still no solution known apart from the obvious ones: $(1,1,1),(4,4,-5),(4,-5,4)$, and $(-5,4,4)$. For $n=30$, the first solution was found by N. Elkies and his coworkers in 2000 [El]. It is interesting to note that, in 1992, D. R. Heath-Brown HB had made a prediction on the density of the solutions for $n=30$ without knowing any solution explicitly.

Over the years, a number of algorithms have been developed in order to attack the general problem. An excellent overview concerning the various approaches invented up to around 2000 was given in BPTY, published in 2007. The historically first algorithm which has a complexity of $O\left(B^{1+\varepsilon}\right)$ for a search bound of $B$ is the method of R. Heath-Brown [HLR. The best algorithm presently known is Elkies' method described in [El].

\section{ELKIES' METHOD}

This algorithm is geometric in nature. The idea is to cover the curve $Y=\sqrt[3]{1-X^{3}}, X \in[0,1 / \sqrt[3]{2}]$, by very small parallelograms which we call flagstones. The algorithm finds all rational points of the particular form $(x / z, y / z)$ which are contained in one of the flagstones for $z \in \mathbb{N}$ up to a given bound $B$. (This means we are searching for triples such that $x^{3}+y^{3}-z^{3}$ will be small.)

For each flagstone, this is equivalent to the detection of all points of the standard lattice $\mathbb{Z}^{3}$ that are contained in a certain pyramid. The problem is that, viewed in the standard coordinates, this pyramid has an enormous height in comparison with the two other dimensions. Thus, it has an extremely sharp apex. Searching naively for lattice points in such a pyramid would be highly inefficient.

Received by the editor February 12, 2008 and, in revised form, April 10, 2008.

2000 Mathematics Subject Classification. Primary 11Y50; Secondary 14G05, 14J28.

Key words and phrases. Diophantine equation, sum of three cubes, Elkies' method.

The computer part of this work was executed on the Sun Fire V20z Servers of the Gauss Laboratory for Scientific Computing at the Göttingen Mathematisches Institut. Both authors are grateful to Professor Y. Tschinkel for permission to use these machines as well as to the system administrators for their support.

(c) 2008 American Mathematical Society 1227

Reverts to public domain 28 years from publication 
The idea to overcome this difficulty is to work in coordinates more adapted to this pyramid. The drawback in this case is that the basis $\{(1,0,0),(0,1,0),(0,0,1)\}$ of the standard lattice is then far from being reduced in whatever sense. One needs to apply lattice basis reduction. Having done that, searching for lattice points within the pyramid is essentially equivalent to a search for small points of the lattice. For this, one may use the well-known algorithm of Fincke-Pohst FP.

The size of the flagstones is somewhat arbitrary. Smaller flagstones mean that more time is required for lattice basis reductions. Larger ones lead to more time being spent on the algorithm of Fincke-Pohst. The optimum size depends on details of the implementation.

\section{IMPLEMENTATION}

Our implementation of Elkies' method is written in C and C++. We took care that only initialization parts of the code were written in $\mathrm{C}++$ or made use of the multi-precision floats of GMP.

The time-critical parts were written in plain C making some use of the instruction asm. It turned out that, for most of the computations, 128-bit fixed-point arithmetic was sufficiently precise. We realized the 128-bit fixed-point numbers as arrays consisting of two long ints. The arithmetic of the fixed-point numbers was implemented in such a way that all loops (of length two) were manually unrolled.

For lattice basis reduction, we implemented a version of LLL for three-dimensional lattices. It turned out that adjacent flagstones led to similar reduced bases. That is why enormous savings could be achieved by doing LLL incrementally. We start the LLL computation for the next flagstone with a reduced basis of the previous one and not with a naive basis.

Another substantial improvement was realized in the Fincke-Pohst part. Here, one has to compute many adjacent values of the same cubic polynomial in three variables. An implementation of a difference scheme reduced most of these computations to a few additions of values obtained before.

Some details. We searched systematically for solutions of $x^{3}+y^{3}+z^{3}=n$ where the positive integer $n<1000$ is neither a cube nor twice a cube and $|x|,|y|,|z|<10^{14}$. The length of the flagstones was chosen dynamically. It was around $8.4 \cdot 10^{-12}$ near $x=0$ and around $6.6 \cdot 10^{-14}$ near $x=1 / \sqrt[3]{2}$. The area of the flagstones was essentially constant at a value near $1.7 \cdot 10^{-40}$. This led to a total number of a bit more than $10^{13}$ flagstones to deal with.

We chose the widths of the flagstones such that all points in a horizontal distance of $<10^{-30}$ from the curve are contained in one of the flagstones. This should make sure that all solutions of heights between $10^{11}$ and $10^{14}$ are certainly found. Indeed, if we arrange variables such that $|x| \leq|y|<|z|$, then the point $(|x / z|,|y / z|)$ is at a horizontal distance from the curve of $\left.\frac{d s^{1 / 3}}{d s}\right|_{s=\left(1-X^{3}\right)} \cdot n /|z|^{3}$, in first order approximation. Since $X:=|x / z|<1 / \sqrt[3]{2}$, the derivative is always less than 0.53 .

The whole search took around ten months of CPU time. Only $14 \%$ of that time was spent on lattice basis reductions. The lion's share was spent searching for small lattice points. I.e., on our implementation of the algorithm of Fincke-Pohst. 


\section{RESUlts}

In comparison with the computations of BPTY] and the lists, dating back to 2001 and published in [Be, 3519 new solutions have been found.

Among them, there are the following. For each of the nine numbers on the left, no solution had been given before, either in D. Bernstein's lists, or in [BPTY].

$$
\begin{aligned}
156 & =26577110807569^{3}-18161093358005^{3}-23381515025762^{3}, \\
318 & =1970320861387^{3}+1750553226136^{3}-2352152467181^{3}, \\
& =30828727881037^{3}+27378037791169^{3}-36796384363814^{3}, \\
366 & =241832223257^{3}+167734571306^{3}-266193616507^{3}, \\
420 & =8859060149051^{3}-2680209928162^{3}-8776520527687^{3}, \\
564 & =53872419107^{3}-1300749634^{3}-53872166335^{3}, \\
758 & =662325744409^{3}+109962567936^{3}-663334553003^{3} \\
& =83471297139078^{3}+77308024343011^{3}-101433242878565^{3}, \\
789 & =18918117957926^{3}+4836228687485^{3}-19022888796058^{3}, \\
894 & =19868127639556^{3}+2322626411251^{3}-19878702430997^{3}, \\
948 & =103458528103519^{3}+6604706697037^{3}-103467499687004^{3} .
\end{aligned}
$$

For 13 values of $n$, for which exactly one solution was known, we found a second one. Among those, there is $n=30$. The second solution for $n=30$ is

$$
30=3982933876681^{3}-636600549515^{3}-3977505554546^{3} .
$$

A second and a third solution for $n=75$ are

$$
\begin{aligned}
75 & =2576191140760^{3}+1217343443218^{3}-2663786047493^{3} \\
& =59897299698355^{3}-47258398396091^{3}-47819328945509^{3} .
\end{aligned}
$$

On the other hand, the highest numbers of solutions found are 93 for $n=792$ and 85 for $n=720$. This fits well with the prediction of $\sim A(n) \log B$ essentially different solutions of height $<B$ made in $[\mathrm{HB}$. Here,

$$
A(n):=\frac{1}{6} \cdot \frac{2}{3} \frac{\Gamma(1 / 3)^{2}}{\Gamma(2 / 3)} \prod_{p} \tau_{p}(n)
$$

for $\tau_{p}(n):=\lim _{k \rightarrow \infty} \frac{N\left(p^{k}\right)}{p^{2 k}}$ where

$$
N\left(p^{k}\right):=\#\left\{(x, y, z) \in\left(\mathbb{Z} / p^{k} \mathbb{Z}\right)^{3} \mid x^{3}+y^{3}+z^{3}=n \in \mathbb{Z} / p^{k} \mathbb{Z}\right\} .
$$

We calculated $A(792) \approx 2.98$ and $A(720) \approx 3.18$ which are the highest values of $A(n)$ for $n<1000$.

A complete list of all 14288 solutions we know for $n<1000, n$ being neither a cube nor twice a cube, is given in EJ]. It was formed by merging together the new solutions, D. Bernstein's lists from 1999 and 2001, and a list of small solutions found by a naive search.

Unfortunately, we still do not know of any solution for $n=33$ or $n=42$. Here, $A(33) \approx 0.089$ and $A(42) \approx 0.113$ would suggest that there could be a few solutions, but not many. More generally, the question whether $x^{3}+y^{3}+z^{3}=n$ has an integral solution remains open for 14 numbers below 1000. These numbers are $33,42,74,114,165,390,579,627,633,732,795,906,921$, and 975 . 


\section{REFERENCES}

[AMD] Software Optimization Guide for AMD Athlon ${ }^{\mathrm{TM}} 64$ and AMD Opteron ${ }^{\mathrm{TM}}$ Processors, Rev. 3.04, AMD, Sunnyvale, CA, 2004.

[BPTY] Beck, M., Pine, E., Tarrant, W., and Yarbrough Jensen, K.: New integer representations as the sum of three cubes, Math. Comp. 76 (2007), 1683-1690. MR2299795 (2007m:11170)

[Be] Bernstein, D.: Threecubes, available at: http://cr.yp.to/threecubes.html.

[El] Elkies, N. D.: Rational points near curves and small nonzero $\left|x^{3}-y^{2}\right|$ via lattice reduction, in: Algorithmic number theory (Leiden 2000), Lecture Notes in Computer Science 1838, Springer, Berlin 2000, 33-63. MR1850598 (2002g:11035)

[EJ] Elsenhans, A.-S. and Jahnel, J.: List of solutions of $x^{3}+y^{3}+z^{3}=n$ for $n<1000$ neither a cube nor twice a cube, available at: http://www.uni-math.gwdg.de/jahnel/ threecubes_20070419.txt.

[FP] Fincke, U. and Pohst, M.: Improved methods for calculating vectors of short length in a lattice, including a complexity analysis, Math. Comp. 44 (1985), 463-471. MR777278 (86e:11050)

[HB] Heath-Brown, D. R.: The density of zeros of forms for which weak approximation fails, Math. Comp. 59 (1992), 613-623. MR1146835 (93a:11055)

[HLR] Heath-Brown, D. R., Lioen, W. M., and te Riele, H. J. J.: On solving the diophantine equation $x^{3}+y^{3}+z^{3}=k$ on a vector computer, Math. Comp. 61 (1993), 235-244. MR 1202610 (94f:11132)

Mathematisches Institut der Universität Göttingen, Bunsenstrasse 3-5, D-37073 Göttingen, Germany

E-mail address: elsenhan@uni-math.gwdg.de

Mathematisches Institut der Universität Göttingen, Bunsenstrasse 3-5, D-37073 Göttingen, Germany

E-mail address: jahnel@uni-math.gwdg.de

URL: http://www.uni-math.gwdg.de/jahnel 